\title{
Antibiotic prescription patterns in primary dental health care in Kosovo
}

NAIM HALITI ${ }^{1, A, D-F}$, SHAIP KRASNIQI ${ }^{2, A, C-F}$, AGIM BEGZATI ${ }^{3, \text { B-D }}$, BASHKIM GLLAREVA ${ }^{4, B-D}$, LUMNIJE KRASNIQ ${ }^{3, \text { B-D }}$, NORA SHABANI ${ }^{4,8-D}$, BLERIM MEHMETI ${ }^{3, \text { B-D }}$, FEHIM HALITI 3 , A-E

${ }^{1}$ Department of Forensic Medicine, University of Prishtina "Hasan Prishtina”, Prishtina, Kosovo

${ }^{2}$ Institute of Pharmacology and Toxicology and Clinical Pharmacology, Faculty of Medicine, University of Prishtina

"Hasan Prishtina", Prishtina, Kosovo

${ }^{3}$ Department of Children Dentistry, University Dentistry Clinical Center of Kosovo, Prishtina, Kosovo

${ }^{4}$ Faculty of Medicine, University of Prishtina "Hasan Prishtina", Prishtina, Kosovo

A - Study Design, B - Data Collection, C - Statistical Analysis, D - Data Interpretation, E - Manuscript Preparation, F - Literature Search, G - Funds Collection

Summary Background. Globally, the level of antibiotic prescription in dental care is increasing each year, and evidence shows a high level of antibiotic misuse.

Objectives. The survey was conducted to determine the antibiotic prescription pattern in primary dental care in Kosovo, to analyze prescription habits, to identify eventual misuses of antibiotics and to facilitate the formulation of standards for the rational prescription of these drugs.

Material and methods. The data of 1,825 registered patients for a 1-year period was randomly collected and analyzed concerning antibiotic use and presented by Defined Daily Doses [DDD]/1,000 inhabitants/day as per the standards of WHO methodology.

Results. The prescription rate of antibiotics in the general number of registered patients was $7.9 \%$. The total consumption of antibiotic drugs in dental primary care was $2.17 \mathrm{DDD} / 1,000$ inhabitants/day. The total number of individual antibiotics during the survey was 6 agents. The most used antibiotic was Co-amoxiclav (J01CR02) with 1.16 DID, followed by Amoxicillin (J01CA04) 0.78 DID. While other individual antibiotics were used significantly less, with Ceftriaxone (J01DD04), with a 0.11 DID, Cefalexin (J01DB01), with a 0.09 DID, Procaine Benzyl Penicillin (J01CE09), with a 0.02 DID, and Gentamicin (J01GB03), with a 0.01 DID.

Conclusions. The results of survey indicate a high and irrational prescription of antibiotics in primary dental care in Kosova. The prescription of broad-spectrum antibiotics may have a negative impact in destroying commensal flora and triggering bacterial resistance. The use of broad-spectrum antibiotic, especially parenteral antibiotics, should be replaced with more narrow-spectrum oral antibiotics, and a more restrictive prescription pattern should be put in place. For the qualitative improvement of prescription of these drug groups, we recommend the implementation of a restrictive antibiotic policy.

Key words: dentistry, primary healthcare, antibiotic use.

Haliti N, Krasniqi S, Begzati A, Gllareva B, Krasniqi L, Shabani N, Mehmeti B, Haliti F. Antibiotic prescription patterns in primary dental health care in Kosovo. Fam Med Prim Care Rev 2017; 19(2): 128-133, doi: https://doi.org/10.5114/fmpcr.2017.67866.

\section{Background}

Antibiotics represent one of the most important drug groups in clinical practice, considering their role in the control of infectious diseases and their impact on public health. Dental care service includes different types of care, such as the treatment of odontogenic infections. Globally, the level of antibiotic prescription in dental care is increasing annually, and antibiotics are used extensively in the field of endodontics $[1,2]$. These prescription habits reflect the trends of overuse and misuse of antibiotics in dental practice. The extensive utilization of antibiotics in clinical practice has been determined to be a leading factor in the emergence of antibiotic resistance [3]. However, the relationship between the antibiotic prescription rate and bacterial resistance is relatively complex. Evidence indicates that antibiotic use influences resistance, but a persuasive, quantitative relationship between the volume of antibiotic use and bacterial resistance has not yet been established [4]. Antibiotic prescription by dental practitioners has an important impact on the rate of general antibiotic prescription use, and an attempt has been made to establish a surveillance system for the monitoring and control of the use of these drugs $[5,6]$.
The rational and effective prescription of antimicrobials is imperative in dental practice, and it is necessary to implement an antimicrobial prescription monitoring system and antibiotic stewardship program. One important strategy for reaching the objective of rational antibiotic prescription is the implementation of drug utilization studies, as defined by the World Health Organization (WHO) [7]. Antibiotic utilization studies enable analyses of antibiotic use, provide feedback data on the distribution of prescriptions and measure the effects of restrictive measures on the level of antibiotic use $[8,9]$. Hence, such studies are considered a fundamental starting point in establishing an effective antibiotic stewardship program, with the main objectives of improving treatment efficacy and decreasing bacterial resistance; this program can also be used as a pharmaco-epidemiological measure for implementation of a national restrictive antibiotic policy [10]. Systematic reviews of antibiotic use have revealed effective measures by integrating the results of studies demonstrating effective restrictive programs and decreased antibiotic use $[11,12]$. Despite systematic monitoring and the extensive antibiotic use programs in developed countries, the data on antibiotic use in most low- and middle-income countries is scarce and insufficient. Specifically, information on antibiotic use in dental practice is widely unavailable. Thus, the use of 
an antimicrobial prescription monitoring system and antibiotic stewardship program will enable the reduction of prescription errors, increase the safety of drugs and reduce the triggering of drug resistance.

\section{Objectives}

The survey was conducted to determine the antibiotic prescription pattern in primary dental care in Kosovo, to analyze prescription habits, to identify eventual misuses of antibiotics and to facilitate the formulation of standards for the rational prescription of these drugs.

\section{Material and methods}

\section{Ethics Statement}

This survey was performed according to the guidelines of the World Medical Association Declaration of Helsinki using an anonymous system of patient data collection. According to the local regulations in Kosovo, health workers who use descriptive surveys may collect data using an anonymous system with committee approval.

\section{Methodology}

This study was conducted in the six administrative regions of primary dental health care in Kosovo. In each region, the dental services of the Main Family Medicine Center (MFMC) and of one other Family Medicine Center (FMC) were included in the survey. In total, the study was conducted in twelve primary dental care centers in Kosovo.

In this retrospective study, from 27,375 patient records found at the register of primary health care centers involved in the study, 1 of every 15 records was chosen for analysis, for a total of 1,825 patient records. We used the dental patient register from the beginning of January to the end of December 2015.

Data was collected manually by our team using an approved protocol for data collection. The members of the collection team attended training on drug utilization.
The indicators specified by the WHO Action Programme on Essential Drugs and the International Network for the Rational Use of Antibiotics were included on the data collection form [12].

The Anatomical Therapeutic Chemical (ATC) Classification System of WHO was applied in this study. The WHO recommends the use of the Drug Utilization Research (DUR) methodology, which includes the Anatomic Therapeutic Classification/ /Defined Daily Dose (ATC/DDD) index [13].

The ATC/DDD index ATC codes and DDD values for each individual drug were registered. We included all antibacterials for systemic use (ATC J01), excluding those used as intestinal anti-infectives (ATCA07AA).

The patient records were assigned a code for identification, and the patient data was collected using an anonymous methodology and entered into an approved Excel spreadsheet. This data included patient age and sex, the diagnosis according to the International Statistical Classification of Diseases and Related Health Problems: $10^{\text {th }}$ Revision (ICD-10) code, the daily dose of the antimicrobial drug used, the antimicrobial classification by ATC codes, the trade or generic name of the drug, the number of antibiotics prescribed to each patient and the antibiotic administration route [13].

In this study, the data was analyzed using Microsoft ${ }^{\circledR}$ Excel software 2007, USA. The results are presented using descriptive statistics, such as the frequency of distribution. Quantitative analysis was performed using a methodology based on DDD/1,000 inhabitants/day and ATC, according to the WHO [14].

\section{Results}

A total of 1,825 patients were included in our survey. Among them, $49.3 \%$ were male, and $50.7 \%$ were female. The gender distributions did not significantly differ across the 6 regions (regions 01 to 06 ).

The prescription rate of antibiotics for the total number of registered patients was $7.9 \%$. The percentages of patients treated with antibiotics varied across the regions, ranging from a low of $4.75 \%$ in region 01 to a high of $12.8 \%$ in region 02 ; in addition, the rates were $10.5 \%$ and $9.2 \%$ in regions 04 and 03 , respectively. Of the 1,825 patients, 87 (4.8\%) underwent surgical interventions, whereas the other 1,738 received pharmacological dental treatment (Table 1).

\begin{tabular}{|l|l|l|l|l|l|l|l|}
\hline \multicolumn{2}{|l|}{ Table 1. General data and quantitative indicators of antibiotic prescription } \\
\hline Region & $\begin{array}{l}\text { Nr. of } \\
\text { patients }\end{array}$ & $\begin{array}{l}\text { Nr. of males } \\
(\%)\end{array}$ & $\begin{array}{l}\text { Nr. of } \\
\text { females (\%) }\end{array}$ & $\begin{array}{l}\text { Nr. of patients } \\
\text { w/antibiotics (\%) }\end{array}$ & $\begin{array}{l}\text { Nr. of patients } \\
\text { wo/antibiotics } \\
(\%)\end{array}$ & $\begin{array}{l}\text { Nr. of patients } \\
\text { with surgical in- } \\
\text { terventions (\%) }\end{array}$ & $\begin{array}{l}\text { Nr. of patients } \\
\text { with nonsurgical } \\
\text { interventions (\%) }\end{array}$ \\
\hline 01 (Prishtina) & 362 & $169(46.7 \%)$ & $193(53.3 \%)$ & $17(4.75 \%)$ & $345(95.3 \%)$ & $17(4.7 \%)$ & $345(95.3 \%)$ \\
\hline 02 (Mitrovica) & 288 & $145(50.3 \%)$ & $143(49.7 \%)$ & $37(12.8 \%)$ & $251(87.2 \%)$ & $8(2.8 \%)$ & $280(97.2 \%)$ \\
\hline 03 (Peja) & 303 & $156(51.5 \%)$ & $147(48.5 \%)$ & $28(9.2 \%)$ & $275(90.8 \%)$ & $7(2.3 \%)$ & $296(97.7 \%)$ \\
\hline 04 (Prizren) & 306 & $154(50.3 \%)$ & $152(49.7 \%)$ & $32(10.5 \%)$ & $274(89.5 \%)$ & $31(10.1 \%)$ & $275(89.9 \%)$ \\
\hline 05 (Ferizaj) & 266 & $129(48.5 \%)$ & $137(51.5 \%)$ & $14(5.3 \%)$ & $252(94.7 \%)$ & $12(4.5 \%)$ & $254(95.5 \%)$ \\
\hline 06 (Gjilan) & 300 & $146(48.7 \%)$ & $154(51.3 \%)$ & $16(5.3 \%)$ & $284(94.7 \%)$ & $12(4.0 \%)$ & $288(96 \%)$ \\
\hline Total & 1825 & $899(\mathbf{4 9 . 3 \% )}$ & $\mathbf{9 2 6 ( 5 0 . 7 \% )}$ & $\mathbf{1 4 4}(\mathbf{7 . 9 \% )}$ & $\mathbf{1 6 8 1}(\mathbf{9 2 . 1 \% )}$ & $\mathbf{8 7}(\mathbf{4 . 8 \% )}$ & $\mathbf{1 7 3 8 ( 9 5 . 2 \% )}$ \\
\hline
\end{tabular}

\begin{tabular}{|c|c|c|c|c|c|c|c|}
\hline Region & $\begin{array}{l}\text { Nr. of an- } \\
\text { tibiotics }\end{array}$ & $\begin{array}{l}\text { Male prescrip- } \\
\text { tion }\end{array}$ & $\begin{array}{l}\text { Female pre- } \\
\text { scription }\end{array}$ & $\begin{array}{l}\text { Generic name } \\
\text { (Nr/\%) }\end{array}$ & $\begin{array}{l}\text { Brand name } \\
(\mathrm{Nr} / \%)\end{array}$ & $\begin{array}{l}\text { Oral antibiotics } \\
\text { (Nr/\%) }\end{array}$ & $\begin{array}{l}\text { Parenteral anti- } \\
\text { biotics ( } \mathrm{Nr} / \%)\end{array}$ \\
\hline 01 (Prishtina) & 17 & $11(64.7 \%)$ & $6(35.3 \%)$ & 6 (35.3\%) & $11(64.7 \%)$ & $17(100 \%)$ & $0(0 \%)$ \\
\hline 02 (Mitrovica) & 43 & 25 (58.1\%) & $18(41.9 \%)$ & 42 (97.7\%) & $1(2.3 \%)$ & $21(48.8 \%)$ & 22 (51.2\%) \\
\hline 03 (Peja) & 30 & $13(43.3 \%)$ & $17(56.7 \%)$ & $29(96.7 \%)$ & $1(3.3 \%)$ & $27(90.0 \%)$ & $3(10 \%)$ \\
\hline 04 (Prizren) & 32 & 15 (46.9\%) & $17(53.1 \%)$ & $12(37.5 \%)$ & $20(62.5 \%)$ & $24(75 \%)$ & $8(25 \%)$ \\
\hline 05 (Ferizaj) & 14 & $7(50.0 \%)$ & 7 (50.0\%) & $10(71.4 \%)$ & $4(28.6 \%)$ & $14(100 \%)$ & $0(0 \%)$ \\
\hline 06 (Gjilan) & 16 & $8(50.0 \%)$ & $8(50.0 \%)$ & $14(87.5 \%)$ & $2(12.5 \%)$ & $16(100 \%)$ & $0(0 \%)$ \\
\hline Total & 152 & 79 (52.0\%) & 73 (48.0\%) & 113 (74.3\%) & 39 (25.7\%) & 119 (78.3\%) & 33 (21.7\%) \\
\hline
\end{tabular}


For the 144 patients who were prescribed antibiotics, 152 antibiotic prescriptions were registered. Comprehensive analyses of antibiotic use revealed that 136 patients were prescribed only 1 antibiotic, whereas 8 received a combination of 2 antibiotics. Of the 8 patients who received 2 antibiotics, 7 received the combination ceftriaxone and gentamicin, and 1 was prescribed the combination of procaine benzylpenicillin and gentamicin.

The antibiotic prescription rate was slightly higher for male patients than for female patients ( $52 \%$ vs. $48 \%$, respectively). In addition, greater differences in this rate between genders were detected among the different regions. Specifically, in regions 01 and 02 , more males than females used antibiotics $(64.7 \%$ and $58.1 \%$, respectively), whereas in regions 03 and 04 , more females than males used them ( $56.7 \%$ and $53.1 \%$, respectively).

Analysis of the prescription of antibiotics by generic name and brand name revealed that generic antibiotics were prescribed significantly more frequently than brand name antibiotics ( $74.3 \%$ vs. $25.7 \%$, respectively). Specifically, in regions 02 and 03, generic antibiotics were prescribed at significantly higher rates of $97.7 \%$ and $96.7 \%$, respectively, compared with brand name antibiotics, whereas the opposite was observed in region 04 , where the prescription rate of brand name antibiotics was $62.5 \%$.

The total percentage of oral antibiotics was significantly higher than that of parenteral antibiotics $(78.3 \%$ vs. $21.7 \%$, respectively). Absolute prescription rates $(100 \%)$ for oral antibiotics were registered in regions 01,05 and 06 , whereas in region 02 , the rate for parenteral antibiotics was higher than that for oral antibiotics ( $51.2 \%$ vs. $48.8 \%$, respectively) (Table 2 ).

Analyses of medical records did not indicate that antibiotic sensitivity testing was conducted for any patient. Therefore, we considered that the prescription of antibiotics was performed empirically.

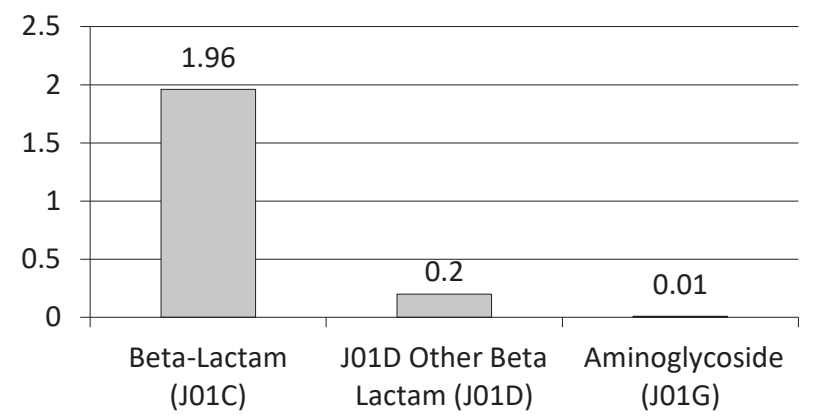

Figure 1. Structure of antibiotic drug use DDD/1,000 inhabitants/day

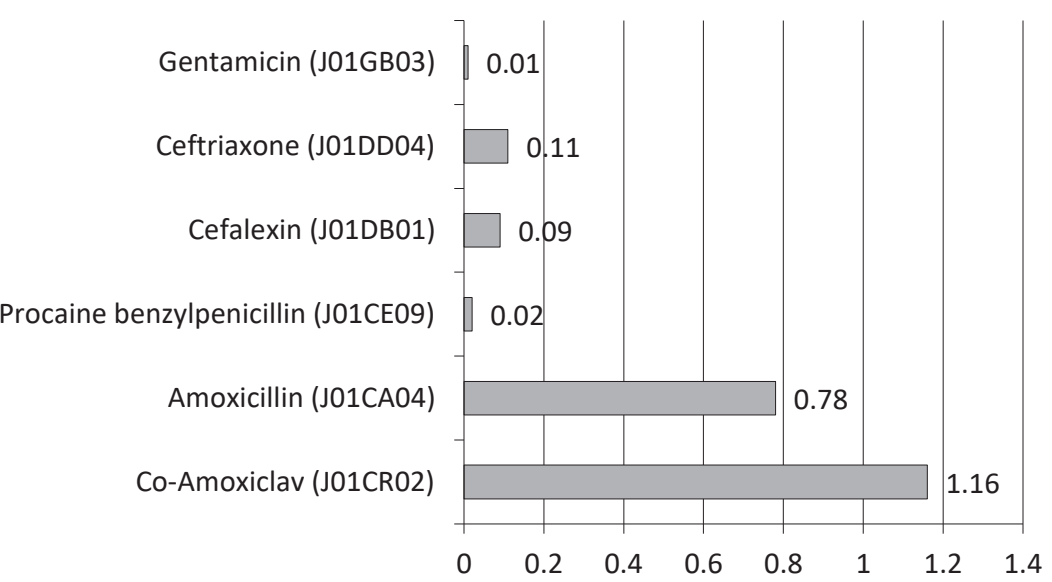

Figure 2. Structure of individual antibiotic DDD/1,000 inhabitants/day
Figure 1 depicts the percentage of utilization for each antibiotic group, presented as DDD/1,000 inhabitants/day (DID). The distribution shows that beta-lactam antibiotics (J01C) were the most frequently used, with a $1.95 \mathrm{DID}$, whereas other beta lactams (J01D) and aminoglycosides (J01G) were used significantly less frequently (with 0.2 and 0.01 DDDs, respectively).

Figure 2 shows the DID values for the 6 antibiotics assessed in this survey. The results showed that dentists in primary dental health care in Kosovo prescribed only 6 individual antibiotics. The most frequently used antibiotic was Co-amoxiclav (J01CR02), with a 1.16 DID, following by Amoxicillin (J01CA04), with a 0.78 DID. Other antibiotics that were used significantly less frequently included Ceftriaxone (J01DD04), with a 0.11 DID, Cefalexin (J01DB01), with a 0.09 DID, Procaine Benzyl Penicillin (J01CE09), with a 0.02 DID, and Gentamicin (J01GB03), with a 0.01 DID.

Overall, 10 classes of diagnosis were recorded for all patients registered in our survey database. The class of diagnosis $\mathrm{K} 08$ (other diseases of teeth and supportive structures) was the most common at $41.2 \%$, followed by class $\mathrm{KO} 4$ (other diseases of hard tissue of teeth) at $37.6 \%$ and $\mathrm{KO2}$ (dental caries) at $10.8 \%$. Other classes of diagnosis, such as K05 (gingivitis and periodontal diseases), KOO (disorders of tooth development and eruption) and K10 (other jaw diseases), were documented less frequently (Figure 3).

During our survey, 6 classes of diagnosis were recorded for which antibiotics were prescribed (Figure 4). The predominant class was $\mathrm{K} 08$ (other diseases of teeth and supportive structures) at $62.5 \%$, followed by K04 (diseases of pulp and periapical tissues) at $28.5 \%$. Other classes were detected at smaller percentages, including $\mathrm{K} 05$ at 2.1\%, A31 (infection due to another mycobacteria) at 1.3\%, K10 (other jaw diseases) and S10 (superficial injuries of teeth). 


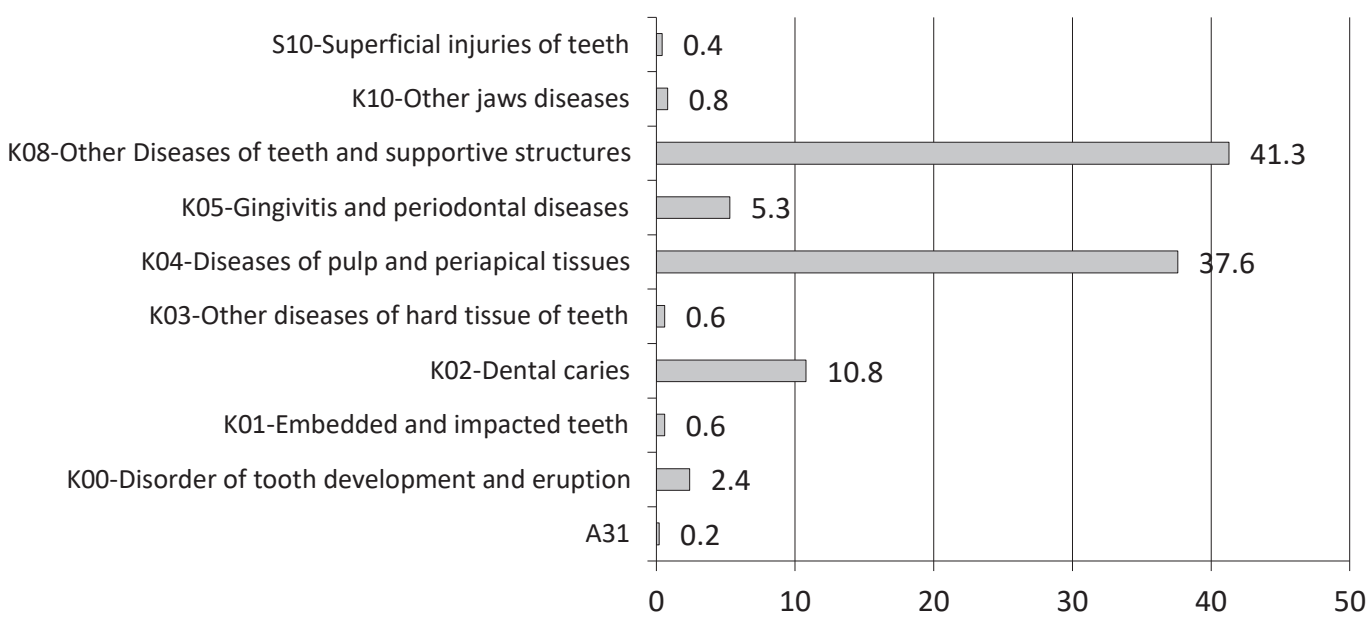

Figure 3. Structure of diagnosis by ICD-10 in total patients $(n=1825)$

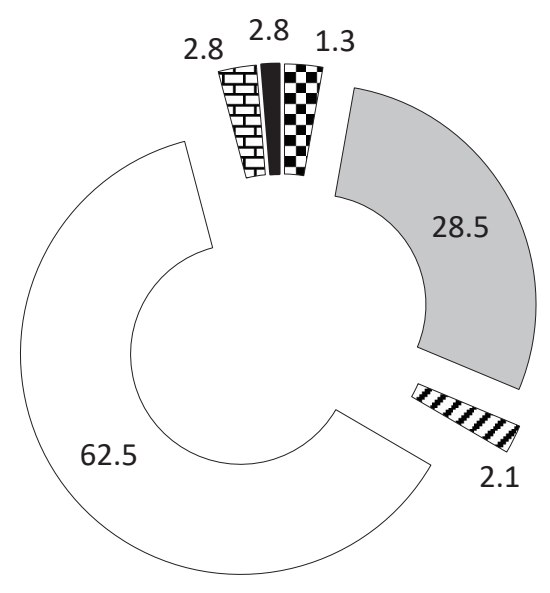

\author{
- A31-Infection due to other \\ mycobacteria \\ $\square$ K04-Diseases of pulp and \\ periapical tissues \\ $\checkmark$ K05-Gingivitis and \\ periodontal diseases \\ $\square$ K08-Other diseases of teeth \\ and supportive structures \\ $\square$ K10-Other jaws diseases \\ S10-Superficial injuries of \\ teeth
}

Figure 4. Structure of diagnosis by ICD-10 in patients with antibiotics $(n=144)$

\section{Discussion}

The surveillance of antibiotic use in primary dental care in Kosovo was a complex and time-consuming process, because the data was manually collected from patient records. As some medical records were not well documented, it was necessary to obtain additional clarification by directly contacting the dentists.

This is the first comprehensive antibiotic surveillance study conducted to assess primary dental care in Kosovo using the WHO methodology for antibiotic use, which is a drug utilization research methodology based on the Anatomic Therapeutic Classification/Defined Daily Dose (ATC/DDD) index [15].

The rate of antibiotic use for the total number of dental patients was $7.9 \%$, which can be considered optimal under the circumstances of this study, because applicable antibiotic treatment guidelines and protocols are not available in Kosovo; thus, there are no restrictions on antibiotic prescription in primary dental care [16]. According to published data, there is an increased trend of antibiotic prescription in dental practice, and Marra et al. presented that the percentage of antibiotic prescriptions by dentists assessed in Canada from 1996 to 2013 increased from $6.7 \%$ to $11.3 \%$ [17].

The total outpatient antibiotic (ATC group J01) use was 2.17 DID and is substantially higher than the prescription of antibiotic by dentist in the Czech Republic (0.75 DID) [2] and in Canada (1.59 DID) [17]. In addition, beta-lactam antibiotics (J01C) were the most frequently used. These antibiotics can be considered a rational choice for use in dental practice, especially narrow- -spectrum penicillins. The total use of this antibiotic group is significantly higher compared with the results of antibiotic prescription in Croatia (89.4\% vs. $69.9 \%)[18,19]$.

Analysis of the different groups of antibiotics revealed that only 3 groups were prescribed, which indicates that the choice of antibiotics by dentists is relatively rational, homogenous and consensual. Furthermore, the evidence obtained from analysis of the prescription rates of the antibiotic groups does not support the prescription of other beta-lactam antibiotics, especially aminoglycosides, without prior antibiotic sensitivity testing [20].

A total of 6 individual antibiotics were recorded in the survey, which is a relatively small number and is attributed to the lack of protocols for the treatment of dental infections in primary dental care. Pipalova et al. presented that in the Czech Republic, there is a decline of narrow-spectrum penicillins by $4.8 \%$, tetracyclines by $3.5 \%$ and macrolides by $3.6 \%$, accompanied by an increasing rate of prescription of co-amoxiclav by $8.9 \%$ and lincosamides by $8.5 \%$ [2].

Interestingly, in our survey, we found no prescription of lincosamides, while there is clinical evidence from general dental practices in Eastern England which support the use of clindamycin in dentistry [21]. From our contact with dental doctors, we revealed that the problematic safety profile of clindamycin with its impact on the destruction of the intestinal bacterial flora of patients is a reason for not prescribing this antibiotic by dentistry doctors. The dentistry doctors did not mention other reasons. Moreover, lincosamides are not in the essential drug list of Ministry of Health for the primary health care level.

The results of the surveys showed that co-amoxiclav, a broad-spectrum antibiotic, was the most frequently prescribed 
drug (with a 1.16 DID) in all regions of Kosovo, and it appears to be the first-line antibiotic for use in the treatment of dental infections. This antibiotic was selected empirically, as the bacterial resistance rate for dental infections in Kosovo was unknown. The rate of prescription of co-amoxiclav was high in contrast with its prescription rates in developed countries. Moreover, Kuriyama et al. have indicated that there are no differences in the clinical outcomes of patients using penicillin $\mathrm{V}$, amoxicillin or a combination of amoxicillin and clavulanate [22].

The second most commonly used antibiotic was amoxicillin, which is considered one of safest empirical antibiotics. It has an appropriate spectrum of activity for oral bacteria and can reach an effective gingival concentration at the site of action [23].

Co-amoxiclav (53.5\%) and amoxicillin (35.9\%) were the most frequently used antibiotics, with a combined prescription rate of $89.4 \%$, whereas the combined prescription rate for the other 4 antibiotics was only $10.6 \%$ of all prescriptions.

Ceftriaxone (J01DD04) was the third most frequently used antibiotic, with a 0.11 DID (5.4\% of the total prescribed antibiotics). Because ceftriaxone is a parenteral, third-generation cephalosporin, its use in primary dental care is not considered rational and is not supported by clinical evidence. This antibiotic is prescribed for the treatment of dental abscess, and its use in primary dental care could trigger bacterial resistance [24].

Cephalexin was the fourth most commonly used antibiotic, with a 0.09 DID (4.1\%), and it is the antibiotic of choice for some dental infections due to its good bone penetration [20].

The use of gentamicin (with a 0.01 DID and $0.5 \%$ prescription rate) is considered less rational due to clinical evidence indicating that it should be reserved for the treatment of select Gram-negative infections, usually occurring in hospital settings [17, 25].
During our survey, 10 classes of diagnosis were identified among the total patients according to the International Classification of Diseases (ICD-10). The most common classes of diagnosis were $\mathrm{K} 08$ (other diseases of teeth and supportive structures) at $41.2 \%, \mathrm{KO} 4$ (other diseases of hard tissue of teeth) at $37.6 \%$ and $\mathrm{KO} 2$ (dental caries) at $10.8 \%$. The most frequent classes of diagnosis for patients taking antibiotics were very similar to those for all registered patients. For the group of patients who received antibiotics, the predominant class of diagnosis was $\mathrm{KO} 8$ (other diseases of teeth and supportive structures) at $62.5 \%$, followed by K04 (diseases of pulp and periapical tissues) at $28.5 \%$. A limitation of the present study is that the data was manually collected from patient medical records.

In general, based on our main findings, we recommend improving the system used for data recording, management and maintenance, which may result in a better and more efficient drug prescription monitoring system in primary dental care.

\section{Conclusions}

Our results have revealed a high prescription rate of antibiotics in primary dental care in Kosovo. The prescription of parenteral antibiotics such as ceftriaxone and gentamicin is not rational and is absolutely non-compliant with protocols for antibiotic use in primary dental care. The use of broad-spectrum antibiotics should be replaced with that of more narrow-spectrum antibiotics, and a more restrictive prescription pattern should be established; therefore, there is an urgent need for the implementation of national guidelines and an antibiotic policy, especially to establish an antibiotic stewardship program for dental care.

Source of funding: This work was funded by the authors' own resources. Conflict of interest: The authors declare no conflict of interests.

\section{References}

1. Falkenstein S, Stein JM, Henne K, et al. Trends in antibiotic use and microbial diagnostics in periodontal treatment: comparing surveys of German dentists in a ten-year period. Clin Oral Investig 2016; 20(8): 2203-2210.

2. Pipalova R, Vlcek J, Slezak R. The trends in antibiotic use by general dental practitioners in the Czech Republic (2006-2012). Int Dent J 2014; 64(3):138-143.

3. Goossens H. Antibiotic consumption and link to resistance. Clin Microbiol Infect 2009; 15: 12-15.

4. Cantón R, Horcajada JP, Oliver A, et al. Inappropriate use of antibiotics in hospitals: the complex relationship between antibiotic use and antimicrobial resistance. Enferm Infecc Microbiol Clin 2013; 31(Suppl. 4): 3-11.

5. World Health Organization (WHO). The role of education in the Rational Use of Medicines. SEARO Techinical Publication Series. WHO, South East Asia; 2006: 45.

6. Finch R. Antibiotic resistance - from pathogen to disease surveillance. Clin Microbiol Infect 2002; 8(6): 317-320.

7. Lu Y, Hernandez P, Abegunde D, et al. The world medicines situation 2011. $3^{\text {rd }}$ ed. Geneva: World Health Organization; 2011.

8. Schmidt-Mende K, Hasselström J. Drug utilization review - a practical example from primary care. Lakartidningen 2013; 110(22): 1105-1107 (in Swedish).

9. Baysari MT, Oliver K, Egan B, et al. Audit and feedback of antibiotic use: utilising electronic prescription data. Appl Clin Inform 2013; 4(4): 583-595.

10. Bachhav SS, Kshirsagar NA. Systematic review of drug utilization studies \& the use of the drug classification system in the WHO-SEARO Region. Indian J Med Res 2015; 142(2): 120-129.

11. Versporten A, Coenen S, Adriaenssens N, et al. European Surveillance of Antimicrobial Consumption (ESAC): outpatient cephalosporin use in Europe (1997-2009). J Antimicrob Chemother 2011; 66(Suppl. 6): vi25-vi35.

12. World Health Organization (WHO). How to investigate drug use in health facilities. Selected drug use indicators. World Health Organization; 1993.

13. World Health Organization (WHO). International statistical classification of diseases and related health problems. World Health Organization; 2009

14. WHO Collaborating Centre for Drug Statistics Methodology. Guidelines for ATC classification and DDD assignment. WHO Collaborating Centre for Drug Statistics Methodology: Oslo; 1996.

15. Monnet DL, Mölstad S, Cars O. Defined daily doses of antimicrobials reflect antimicrobial prescriptions in ambulatory care. J Antimicrob Chemother 2004; 53(6): 1109-1111.

16. Dar-Odeh NS, Abu-Hammad OA, Al-Omiri MK, et al. Antibiotic prescribing practices by dentists: a review. Ther Clin Risk Manag 2010; 6: 301-306.

17. Marra F, George D, Chong M, et al. Antibiotic prescribing by dentists has increased: Why? J Am Dent Assoc 2016; 147(5): $320-327$.

18. Perić M, Perković I, Romić M, et al. The pattern of antibiotic prescribing by dental practitioners in Zagreb, Croatia. Cent Eur J Public Health 2015; 23(2): 107-113.

19. Rachmawati M, Yoshida N, Tsuboi H. Investigation of antibiotic use at a Dental Teaching Hospital in Yogyakarta, Indonesia: a review from guidelines. Pharmacology \& Pharmacy 2014; 5(5): 524-531. 
20. Ramu C, Padmanabhan TV. Indications of antibiotic prophylaxis in dental practice - review. Asian Pac J Trop Biomed 2012; 2(9): 749$-754$.

21. Chate RAC, White S, Hale LRO, et al. The impact of clinical audit on antibiotic prescribing in general dental practice. Br Dent J 2006; 201(10): 635-641.

22. Kuriyama T, Absi EG, Williams DW, et al. An outcome audit of the treatment of acute dentoalveolar infection: impact of penicillin resistance. Br Dent J 2005; 198(12): 759-763.

23. Tenenbaum H, Jehl F, Gallion C, et al. Amoxicillin and clavulanic acid concentrations in gingival crevicular fluid. J Clin Periodontol 1997; 24(11): 804-807.

24. Pasquantonio $\mathrm{G}$, Condò S, Cerroni L, et al. Antibacterial activity of various antibiotics against oral streptococci isolated in the oral cavity. Int J Immunopathol Pharmacol 2017; 25(3): 805-809.

25. Poveda Roda R, Bagan JV, Sanchis Bielsa JM, et al. Antibiotic use in dental practice. A review. Med Oral Patol Oral Cir Bucal 2007; 12(3): E186-E192.

Tables: 2

Figures: 4

References: 25

Received: 16.01 .2017

Revised: 19.02.2017

Accepted: 28.02.2017

Address for correspondence:

Fehim Haliti, MD, PhD

Departament of Children Dentistry

University Dentistry Clinical Center of Kosovo

Prishtina

Kosovo

Tel.: +381 385006002012

E-mail: fehim.haliti@uni-pr.edu 